\title{
Zentrale Ergebnisse und Empfehlungen der interdisziplinären Arbeitsgruppe
}

Ortwin Renn/Uwe Pfenning

unter Mitwirkung von Carl Ludwig Holtfrerich/Heinz Duddeck/Randolf Menzel

Dieser Abschnitt fasst die zentralen Ziele, Ergebnisse und Empfehlungen der interdisziplinären Arbeitsgruppe Zur Zukunft technischer und naturwissenschaftlicher Bildung in Europa der Berlin-Brandenburgischen Akademie der Wissenschaften zusammen. Diese Stellungnahmen und Empfehlungen wurden vom Rat der BBAW auf seiner Sitzung vom 1. Dezember 2011 nostrifiziert.

Die zentralen Ziele der MINT-Bildung sind:

a) die Vermittlung einer fundierten MINT-Kompetenz zum Verständnis der elementaren Vorgänge in Natur und Technik und zur Bewertung der sozialen, wirtschaftlichen und kulturellen Folgen von wissenschaftlichen Erkenntnissen und technischen Innovationen (Wissenschafts- und Technikmündigkeit; im englischen Sprachraum: scientific and technical literacy). Dieser Bildungsauftrag zielt darauf ab, Kinder und Jugendliche mit ihrer wissenschaftlich-technisch geprägten Umwelt vertraut zu machen und sie zu befähigen, gesellschaftliche Zusammenhänge und Folgen wie Chancen, Risiken und mögliche gesellschaftliche Veränderungen kompetent beurteilen zu können.

b) eine frühzeitige und kontinuierliche Förderung begabter junger Menschen, damit sie ihre Neigungen und Fähigkeiten auch im Verlauf ihrer Ausbildung und ihrer beruflichen Karriere erkennen und entfalten können (Talentförderung). Dieser Bildungsauftrag zielt darauf ab, Förderungsprogramme anzubieten, um talentierte Jugendliche für MINT-Ausbildungswege und Berufe optimal vorzubereiten und so zu motivieren, dass sie diese berufliche Laufbahn auch einschlagen.

Beide Ziele setzen unterschiedliche und differenzierte Methoden der MINT-Bildung voraus. Während die Bildung zur Wissenschafts- und Technikmündigkeit auf anschauliche und lebensweltlich nachvollziehbare Bildungsinhalte besonderen Wert legt und sich stärker auf die Ausbildung von Urteilsfähigkeit konzentrieren muss, beruht die Talentförderung auf einem kontinuierlichen Angebot von Fördern und Fordern. Beide Formen der MINT-Bildung sind parallel in den Bildungsprogrammen zu verankern. 


\section{Fachkräftemangel in den MINT-Berufen}

Der Fachkräftemangel in den MINT-Berufen wird sich in den nächsten Jahren verschärfen, wenn es nicht gelingt, diese Berufe und die damit verbundenen Ausbildungswege für junge Menschen attraktiver zu gestalten. Die wesentlichen Gründe für diesen Mangel in Deutschland liegen in:

- einer zurückgehenden Zahl junger Menschen an der Gesamtbevölkerung und der Überalterung bei erwerbstätigen Ingenieurinnen und Ingenieuren (demographische Effekte),

- der Ausweitung der Berufsfelder und Kompetenzen in Unternehmen und öffentlichen Verwaltungen für Absolventen von MINT-Studienfächern (Ausbreitungseffekt),

- der zunehmenden Forschungstätigkeit in Wissenschaft und Unternehmen angesichts dynamischer Produktzyklen und innovativer Systemwechsel (z.B. Umweltenergien, E-Mobility, High Definition TV, Liquid Crystal Display Technik u.v.a.) sowie konjunktureller Effekte,

- einer negativen Bilanz bei der Migration von Fachkräften sowie

- einer geringen Zahl talentierter junger Menschen, die sich für eine MINT-Ausbildung entschließen (mangelnde Attraktivität von MINT-Ausbildungsgängen und -Berufen).

Der Fachkräftebedarf kann aus dem nationalen Bestand an MINT-Absolventen nicht mehr gedeckt werden. Dies gilt bereits für den Ersatzbedarf. Um zu einer nachhaltigen Sicherung von Humanressourcen zu kommen, reichen betriebswirtschaftliche und politische Ansätze nicht aus (GreenCard, BlueCard, Verbesserung der Einwanderungsbedingungen für ausländische Fachkräfte). Vielmehr sind intergenerative, integrierende und nachhaltig wirksame Ansätze gefragt. So ist es eine vordringliche Aufgabe, den hier lebenden Menschen mit Migrationsbiographien sowie technisch-naturwissenschaftlich interessierten Frauen verstärkt $\mathrm{Zu}$ gänge zu den MINT-Bildungswegen und -Berufen zu eröffnen.

\section{Empfehlungen}

Aus dem Verständnis heraus, dass MINT-Bildung integraler Bestandteil der Allgemeinbildung seinund die Aufgabe der Talentförderung für junge Menschen mit besonderer Begabung bzw. Neigung für diese Fächergruppe übernehmen soll, hat die interdisziplinäre Arbeitsgruppe Zur Zukunft technischer und naturwissenschaftlicher Bildung in Europa folgende zentrale Empfehlungen ausgearbeitet: 


\section{a) MINT-Bildung interdisziplinär ausrichten}

Eine erfolgreiche MINT-Bildung erfordert ein neues integratives Verständnis, bei dem die tradierten Naturwissenschaften, die neuen Technologien und die mathematischen Grundlagen im Rahmen der MINT-Bildung miteinander verbunden werden. Diese Forderung nach einer integrativen MINT-Bildung reflektiert einen soziokulturellen Wandel im Verständnis von Natur- und Technikwissenschaften als zwei sich ergänzenden Komponenten im Spannungsverhältnis von Erkenntnis- und Gestaltungsinteressen. Deshalb ist bei den Lehrplänen und bei der Unterrichtsgestaltung auf einen interdisziplinären Ansatz und auf eine Integration von natur-, sozial- und geisteswissenschaftlichen Aspekten bei der Behandlung von Naturphänomenen und von Gestaltungsoptionen durch Technik zu achten.

b) MINT-Bildung in der Schule neu aufstellen

Im schulischen Unterricht bedarf es der Einführung einer Unterrichtsform, die vor allem auf ,inquiry-based learning" setzt und hierfür pädagogisch, didaktisch sowie fachlich hochqualifizierte Lehrkräfte einsetzt. Die Lehrkräfte sind in der Anwendung dieser neuen, selbstbestimmten Lernweisen zu schulen. Zusätzlich benötigen die Schulen hierfür die entsprechenden Materialien und gut ausgestattete Fachräume. Für Fortbildung und Ausstattung sind von den Kultusministerien die notwendigen Mittel bereitzustellen. Das „inquiry-based learning" benötigt nicht mehr Unterrichtsstunden als die bisherige lehrerzentrierte Unterrichtsform. Es eignet sich besonders gut für die Ganztagsschule, weil Schülerinnen und Schüler bei dieser Lernform auch eigenständig und selbstorganisiert in den Nachmittagsstunden üben und vertiefen können.

c) Spezielle Angebote zur Talentförderung integrieren

Für die Talentförderung sind Arbeitsgemeinschaften oder Vertiefungskurse anzubieten, die zum einen komplexere und anspruchsvollere Aufgaben an die Lernenden stellen und zum anderen die Jugendlichen bei der eigenständigen Aufarbeitung des Materials und der Eigenentwicklung von Technik unterstützen. Hier empfiehlt sich auch die aktive Teilnahme an den vielen Wettbewerben (etwa bei „Jugend forscht“), um eine hohe Motivation für außergewöhnliche Leistungen zu erzeugen.

d) Berufsnahe Praktika anbieten

Zusätzlich sollten den Jugendlichen Möglichkeiten zu Praktika in Unternehmen und anderen Organisationen vermittelt werden. Die Erforschung der Berufsbilder unter Jugendlichen hat deutlich gezeigt, dass der Alltag von Ingenieuren und Naturwissenschaftlern in Wirtschaft, Verwaltung und Forschung meist mit veralteten Stereotypen wahrgenommen wird (z.B. dass der Berufsalltag wenig kommunikativ geprägt sei). Praktika vor Ort können helfen, bestehende Vor- 
urteile über MINT-Berufe abzubauen, indem die Jugendlichen mehr über die Realität dieser Berufe erfahren.

e) Außerschulische Bildungsangebote vernetzen

Deutschland bietet im Vergleich der europäischen Länder ein breites Angebot an außerschulischen MINT-Initiativen und -Programmen an. Die Effizienz dieses außerschulischen Angebots ist aber noch zu gering. Das liegt vor allem an der fehlenden Vernetzung und der mangelnden Kontinuität der Angebote. Zudem findet nur gelegentlich eine Verzahnung mit den schulischen Aktivitäten und Lehrinhalten statt. Hier ist dringend anzuraten, dass sich die außerschulischen Bildungsträger auf eine gemeinsame Leitlinie von aufeinander bezogenen Inhalten und einander ergänzenden Lernformen verständigen, indem sie Programme und Initiativen koordinieren und absprechen, ein kontinuierliches Bildungsangebot über alle Altersstufen sicherstellen und für eine Anschlussfähigkeit an die schulischen Bildungsinhalte sorgen.

f) Talentförderung und Förderung der Wissenschafts- und Technikmündigkeit trennen

Die außerschulischen Bildungsangebote sollten sich entweder auf den Aspekt der Verbesserung der Wissenschafts- und Technikmündigkeit oder auf die Talentförderung konzentrieren. Beide Ziele lassen sich nicht mit den gleichen Angeboten erreichen. Um das Interesse an MINT-Themen zu wecken und anzuregen, sind Programme vor allem im Kindergarten und in der Grundschule notwendig. Davon gibt es jetzt noch zu wenige. Zur weiteren Förderung talentierter junger Menschen sind Programme ab dem 12. Lebensjahr zielführend. Zurzeit sind die meisten außerschulischen Initiativen auf die Oberstufe der Gymnasien fokussiert. Für diese Altersgruppe kommt die Förderung der Wissenschafts- und Technikmündigkeit zu spät, und selbst für die Talentförderung ist ein früherer Beginn nachweislich effektiver.

g) Bildungsbarrieren abbauen

Trotz vieler Bemühungen bestehen weiterhin noch viele Barrieren für bestimmte Bevölkerungsgruppen in Deutschland, den ihnen angemessenen Bildungsweg einschlagen zu können. Außerdem gibt es Ungleichheiten im Leistungsentgelt oder bei den Karrierechancen. Für MINT-Berufe gilt es deshalb, einerseits gezielte Fördermaßnahmen für Frauen aufzulegen, andererseits sollten Programme für lMenschen mit Migrationsbiographien entwickelt werden. Solche Maßnahmen können auch kurzfristig die Nachwuchsprobleme in der Wirtschaft vermindern helfen. Zugleich werden damit soziale Gerechtigkeit und soziale Integration gefördert.

h) Bildungsinitiativen evaluieren

Für eine integrative MINT-Bildung ist es wichtig, die wissenschaftlich gebotenen Lernziele mit modernen Unterrichtsmethoden, angemessenen Inhalten 
und altersgerechten Lernformen in Einklang zu bringen. An dieser Aufgabe könnten unter anderem auch die Akademien mitwirken. Die Begleit- und Evaluationsforschung ist weiter auszubauen, um unterschiedliche Konzepte für eine erfolgversprechende Kombination aus klassischer Wissensvermittlung, eigenständigem Lernen, Motivationsförderung und praktischer Umsetzung zu überprüfen und empirisch gesicherte Empfehlungen zu erarbeiten.

i) Beteiligungsoptionen weiterentwickeln

Innerhalb und außerhalb der Bildungsinstitutionen sind gesellschaftliche Dialoge über die Chancen, Risiken und Folgen von wissenschaftlichen Erkenntnissen und technologischen Innovationen für die Gesellschaft zu organisieren, um auf der einen Seite die Bedingtheit unserer Kultur durch wissenschaftliche und technische Leistungen bewusst zu machen und auf der anderen Seite die Freiräume für ihre Ausgestaltung aufzuzeigen.

\section{Ausgangslage}

Die technisch-naturwissenschaftliche Bildungslandschaft in Europa ist trotz ihres organisatorischen und inhaltlichen Variantenreichtums von ähnlichen strukturellen Problemlagen geprägt: eine geringe Attraktivität technischer und naturwissenschaftlicher Bildungsangebote, nachlassendes Interesse der Jugendlichen an den MINT-Berufen (Mathematik, Informatik, Natur- und Technikwissenschaften)bis hin zum Fachkräftemangel, vor allem in den klassischen Ingenieurwissenschaften, sowie ein hoher Anteil älterer Ingenieure in der Erwerbsstruktur, die demnächst ersetzt werden müssen. Die Unterschiede in den demographischen und strukturellen Rahmenbedingungen der einzelnen Länder (z.B. Studierendenquote) verändern zwar die Ausgangslage, die jeweiligen Entwicklungstrends sind jedoch fast identisch. So sind die für ihre effektive Früherziehung bekannten skandinavischen Länder wie Norwegen, Dänemark und eingeschränkt Finnland ebenso von Nachwuchsproblemen in den MINT-Fächern betroffen wie Großbritannien mit seinem hohen Anteil an Museen als Teil der nationalen Bildungskultur und intensiven Aktivitäten im Bereich „Public Understanding of Science and Humanities“ (PUSH).

Gegenwärtig steht jedoch politisch wie öffentlich vor allem der Fachkräftemangel in technischen, naturwissenschaftlichen und einigen weiteren akademischen Berufen (z.B. Medizin) im Vordergrund des Interesses. Die Lage bei den Ingenieuren ist besonders problematisch. Im April 2011 waren bundesweit 90.400 offene Stellen für Ingenieure zu besetzen. Die Vakanzen für Maschinen- und Fahrzeugbauingenieure machen hierbei den größten Anteil der offenen Ingenieurstellen aus, dicht gefolgt von den offenen Stellen für Elektroingenieure. Im gleichen Zeitraum waren ca. 23.000 arbeitslose Ingenieure zu verzeichnen. Daraus ergibt sich 
eine Ingenieurlücke von ca. 69.000 Personen. Im Vergleich zum April 2010 hat sich der Bedarf somit fast verdoppelt. Dass der Arbeitsmarkt für Ingenieure „leergefegt" ist, zeigt vor allem der extreme Rückgang der Arbeitslosigkeit von einem stabilen Sockel bis 2005 von über 60.000 auf unter 25.000 Personen im Jahresmittel 2011. Mittel- und langfristig führt zudem die demographische Entwicklung zu weiteren Engpässen auf dem Arbeitsmarkt für Ingenieurinnen und Ingenieure. Allein der jährliche demographische Ersatzbedarf wird nach Berechnungen des VDI in den kommenden Jahren kontinuierlich ansteigen, und zwar bis 2012 auf ca. 36.000 neue Ingenieure jährlich, von 2013 bis 2017 sogar auf fast 40.000, für den Zeitraum 2023 bis 2027 geht der VDI von einem jährlichen Ersatzbedarf von 48.300 Ingenieuren aus (vgl. BfA 2007, 2009, OECD 2008c, 2010a, FEANI 2010, VDI 2011). Die international zu beobachtende Tendenz zur Höherqualifizierung in den MINT-Berufen und der Ausweitung des Berufsfeldes auf nichttechnische Bereiche wie Vertrieb und Service hat nicht nur im Hochschulbereich, sondern auch im Handwerk und in der Industrie zu einem Fachkräftemangel geführt (Eckerle/Weidig/Limbers 2002, Hampel et al. 2003, Impuls-Stiftung et al. 2005, Egeln/Heine 2005, Frietsch/Gehrke 2005, Minks 2005,VDI 2007, 2011, Eurostat 2008a, VDE 2010).

Die bundesweite Ingenieurlücke ist nur ein, wenn auch dramatischer Hinweis auf die mangelnde Attraktivität der MINT-Berufe in Deutschland und in Europa. Für den Informatikbereich zeichnet sich eine ähnlich dramatische Entwicklung ab. Im Bereich der Mathematik, die Biologie und Chemie ist das Problem weniger stark ausgeprägt, die „,ingenieurnahe“ Physik hingegen betrifft es stärker. Die Summe der durch diesen Fachkräftemangel entgangenen Industrieaufträge wird jährlich auf ein mehrfaches Milliardenvolumen beziffert. Der Beschäftigungseffekt von Ingenieurstellen ist zudem besonders hoch, man rechnet pro Ingenieurstelle mit 1.8 bis 4.2 zusätzlichen Stellen in Produktion oder Dienstleistungssektor (VDE 2010, VDI 2011). Als Fazit ist festzuhalten, dass dieser Fachkräftemangel zu einem großen Risiko für die exportorientierte deutsche Volkswirtschaft geworden ist.

\section{Ziele der MINT-Bildung}

Ziel der MINT-Bildung ist zunächst die Erhöhung des Interesses von Jugendlichen an MINT-Ausbildungswegen und an entsprechenden Berufskarrieren. Darüber hinaus ist es aber ebenfalls ein wichtiges Ziel, junge Menschen mit ihrer Umgebung und den Wandlungsprozessen in ihrer Umwelt vertraut zu machen. Dazu gehören vor allem ein basales Verständnis der Naturwissenschaften sowie eine grundlegende Einsicht in die Funktion und kulturelle Bedingtheit von Technik. Hierin liegt auch der Schlüssel für die Entwicklung einer prinzipiellen Offenheit gegenüber den 
wissenschaftlichen und technischen Neuerungen. Unsere Kultur ist maßgeblich durch das Wissen über grundlegende Erkenntnisse der Natur- und Technikwissenschaften und durch die Erfahrung von Gestaltungsvorgängen mittels Technik geprägt. Dies betrifft alle Menschen, die in Bildungs- und Lernprozessen eingebunden sind, unabhängig davon, ob sie einen MINT-Beruf ausüben möchten oder nicht.

Der in diesem Zusammenhang eingeführte Ausdruck „,scientific literacy“ bedeutet deshalb nicht nur Faktenwissen, sondern umschreibt die Vertrautheit eines Menschen mit einer von Wissen und Technik geprägten Lebenswelt. Diese Vertrautheit als Element einer kollektiven Identität zu schaffen und zu fördern ist vorrangige Aufgabe der MINT-Bildung. Diese Sichtweise von Naturwissenschaft und Technik legt den Grundstein für eine Bildung, deren Ziel nicht vordringlich in der Vermittlung von Sachwissen, sondern in der Gewinnung von Kompetenz und Mündigkeit im Alltag und im Berufsleben liegt. Nicht zuletzt ist es Aufgabe der MINT-Bildung, die Menschen in die Lage zu versetzen, sachkundig und souverän die wissenschaftlichen und technischen Debatten unserer Zeit zu verstehen und kompetent dazu Stellung zu nehmen. Wenn Technik und Naturwissenschaften zu sehr auf die funktionalen und ökonomischen Leistungen begrenzt werden, wird das Wahrnehmungsfeld verengt. Unter diesen Umständen werden wissenschaftlichtechnische Lösungen in der Bevölkerung nur noch als Instrumente für Produktion und Konsum eingestuft. Technik und Wissenschaft sind aber mehr: Sie durchdringen den Alltag, beeinflussen gesellschaftliches Leben und verändern die Gesellschaftskultur.

Wissenschafts- und Technikmündigkeit als Bildungsaufgabe hat in vielen Staaten erst begonnen. Die Ausformulierung und Umsetzung dieser Zielsetzungen in konkrete Bildungs- und Lehrpläne und in Studienordnungen befindet sich noch in den Anfängen und sollte Schwerpunkt der weiteren Bildungspolitik sein. Die Vermittlung von Wissenschafts- und Technikmündigkeit ist Voraussetzung für die Gewinnung von sozialer Identität in einer von Wissenschaft und Technik geprägten Gesellschaft. Je mehr Innovationen die moderne Gesellschaft prägen werden, desto wichtiger wird diese Bildungsaufgabe. Auf dieser Basis kann dann auch sachlich fundiert und ethisch reflektiert ein Diskurs über Chancen und Risiken technischer Innovationen geführt werden.

Ergebnisse der Recherchen der interdisziplinären Arbeitsgruppe der BBAW

MINT-Bildung im internationalen Vergleich

Bei der Darstellung der Ergebnisse ist zwischen dem Ziel der Talentförderung (mit dem sekundären Ziel, mehr Jugendliche für MINT-Ausbildungswege und -berufe 
zu gewinnen) und der Entwicklung einer Wissenschafts- und Technikmündigkeit (mit den sekundären Zielen, Interesse zu wecken, Grundkompetenz zu fördern und Urteilsfähigkeit zu verbessern) zu differenzieren. So ist z.B. in Finnland nach Ergebnissen der ROSE-Studie (Sjøberg/Schreiner 2005) das Interesse an technischen Berufen im europäischen Vergleich besonders gering ausgeprägt, obwohl die Leistungen der Schülerinnen und Schüler in diesem Land Spitzenwerte erreichen. Norwegen und Dänemark, die einen reformierten MINT-Unterricht eingeführt haben, vermögen zwar die Schülerinnen und Schüler stärker für MINT zu begeistern als etwa Deutschland oder Frankreich, dennoch gibt es auch dort einen akuten Fachkräftemangel in den meisten MINT-Berufen.

Im Hinblick auf die Wissenschafts- und Technikmündigkeit (OECD 2008a, 2009a, b) schneiden im internationalen Vergleich vor allem jene Staaten gut ab, die ihre MINT-Bildung in den 1990er Jahren auf Basis neuer Erkenntnisse der Lernforschung reformiert haben (Skandinavien, Osteuropa). Die Reformen betrafen die Einführung von mehr selbstbestimmten Unterrichtsanteilen, eine Verstärkung der Praxis- und Projektorientierung, eine stärkere Hinwendung zu neuen Lernformen (inquiry based learning) sowie die Ausbildung interdisziplinär kompetenter Erzieherinnen und Erzieher (oftmals als akademischer Beruf) und Lehrkräfte. Andere Staaten, die ähnlich wie Deutschland eine inkrementelle Optimierungsstrategie verfolgen, haben bei den internationalen Vergleichen zu Wissenschafts- und Technikmündigkeit weniger erfolgreich abgeschnitten. Frankreich, Spanien oder Italien haben ähnliche Probleme bei der MINT-Bildung wie Deutschland (vgl. Heidenreich 1992, Grelon/Stück 1996, Frietsch/Gehrke 2005). In Großbritannien wurde durch die PUSH-Programme sehr viel in die allgemeine MINTAusbildung investiert, dennoch zeigen die vergleichenden Ergebnisse von PISA oder den OECD-Studien eine insgesamt geringe Wirkung.

In Bezug auf die Talentförderung sind die Ergebnisse weniger eindeutig. Die üblichen OECD-Indikatoren wie Bildungsausgaben (absolut oder pro Kopf), Anteil am Bruttoinlandsprodukt (BIP), Verweildauer im Schulsystem (ISCED-Indikatorensystem) usw. erweisen sich nicht als die entscheidenden Größen, wenn es um die Frage der Wirksamkeit der Talentförderung und Attraktivität von MINT-Ausbildungswegen oder Berufen geht. Deutschland befindet sich im internationalen Ländervergleich bei den auf das Bruttosozialprodukt bezogenen relativen Bildungsausgaben im mittleren Bereich. In absoluten Zahlen oder auch bei den Bildungsausgaben pro Kopf steht Deutschland dagegen im europäischen Spitzenfeld. Dennoch ist die Zahl der Absolventen von MINT-Fächern in Deutschland bis 2009 rückläufig gewesen und steigt erst jetzt langsam wieder an. Ein Zusammenhang zwischen Bildungsausgaben pro Kopf und Anzahl der Jugendlichen, die sich für ein MINT-Studium entscheiden, besteht auch nicht in den anderen europäischen Ländern. Für den Erfolg der Talentförderung sind demographische Faktoren, struk- 
turelle Gründe und vor allem der Grad der erreichten technisch-wissenschaftlichen Modernisierung von maßgeblicher Bedeutung.

In einigen Staaten (z.B. Frankreich) lässt sich der Rückgang der Studienanfänger nahezu vollständig auf die Geburtenrückgänge zurückführen. In anderen Staaten sind die Zyklen der wirtschaftlichen Entwicklung, die besonders bei MINT-Fächern weltweit zu hohen Fluktuationen in der Nachfrage führen, und die mangelnde Bereitschaft junger Menschen, die von ihnen selbst als schwierig angesehenen Fächer zu studieren (z.B. USA, UK), wichtige Einflussfaktoren. Ebenso sind die unterschiedlichen Studierendenquoten eines Jahrgangs zu berücksichtigten. In Skandinavien sind diese sehr hoch (über $70 \%$ ), in Frankreich und Großbritannien im OECD-Durchschnitt, in Deutschland ebenso wie in vielen südeuropäischen Staaten weiterhin unterdurchschnittlich.

Besonders wichtig ist jedoch der in einem Land erreichte Grad der Modernisierung und des Wohlstands. Je mehr Modernisierungsbedarf eine Gesellschaft hat, desto höher ist tendenziell das Prestige wissenschaftlich-technischer Berufe, und desto größer ist auch die Attraktivität, diese Berufe für die eigene Karriere zu wählen. Insofern findet man proportional mehr Absolventen von MINT-Studiengängen in den europäischen Ländern, in denen ein hoher Bedarf an Modernisierung besteht (etwa Portugal oder die meisten osteuropäischen Länder). Dies gilt in noch stärkerem Maße für Schwellenländer wie China oder für die meisten arabischen Staaten, in denen MINT-Berufe soziale Aufstiegsmöglichkeiten versprechen.

Dennoch ist die individuelle Talentförderung als Mittel zur Erhöhung der Attraktivität von MINT-Ausbildungswegen nicht irrelevant. Ihr Einfluss lässt sich allerdings in den makrostatistischen Auswertungen nicht nachweisen. Dagegen weisen Untersuchungen über individuelle Lebens- und Karriereverläufe sehr wohl darauf hin, dass viele Jugendliche MINT-Studiengänge gewählt haben, weil sie in Elternhaus, Schule oder in außerschulischen Programmen intensiv gefördert wurden (acatech/VDI 2009). Erfolgreich war diese Talentförderung immer dann, wenn sie frühzeitig begonnen, kontinuierlich fortgesetzt wurde und auf die individuellen Bedürfnisse abgestimmt erfolgte.

\section{MINT-Bildung in Deutschland}

Eine gute Übersicht zum Stand der MINT-Bildung an deutschen Schulen geben Hartmann et al. (2006), insbesondere im Hinblick auf die Berücksichtigung technischer Bildung in den Lehr- und Bildungsplänen. Für Deutschland belegen die PISA-Studien vor allem Defizite im Anwendungswissen, weniger Defizite im abstrakten Fachwissen (OECD 2010e, f). Sie verweisen zudem auf relevante soziale Unterschiede im deutschen Bildungssystem, weil hier der Bildungserfolg stark von 
der sozialen Herkunft abhängig ist (OECD 2010d, Schölling 2005), vor allem erscheinen Migrationsaspekte bedeutsam (OECD 2010c).

Aufgrund der sozialen, aber auch der sprachlichen Barrieren trifft man in Deutschland bisher auch nur wenige junge Menschen mit Migrationsbiographien in MINT-Berufen an. Ebenso ist der Frauenanteil in den klassischen Ingenieurdisziplinen Maschinenbau und E-Technik wie auch in der Physik an deutschen Hochschulen besonders gering (vgl. Godfroy-Genin 2010, Eurostat 2008b, Pfenning/ Renn/Mack 2002). In Nischenstudiengängen mit sozialen Bezügen (Biomedizin, Medizintechnik, Umwelttechnik) finden sich dagegen überdurchschnittlich hohe Frauenanteile. Hinzu kommt ein mehrfacher „Drop-Out“ während und nach dem Studium sowie im Beruf (geschätzt arbeiten ca. 40.000 bis 60.000 Ingenieurinnen nicht im studierten Beruf, Pfenning/Renn/Mack 2002, Ihsen et al. 2009, Pfenning/ Hiller/Renn 2011).

Die geringe Attraktivität der MINT-Fächer für junge Frauen in Deutschland beruht im Wesentlichen auf drei Gründen: einer geringeren Förderung von technisch talentierten Mädchen in Elternhaus und primären Bildungsinstitutionen, einer höheren Abbruchquote im Studium bzw. einem Wechsel in andere Berufe nach dem Abschluss und den geringeren Aufstiegschancen im Beruf (Pfenning et al. 2002/2003, Ihsen et al. 2009). Hinzu kommen Vorurteile und wahrgenommene Diskriminierungen. Nach den Studien des Nachwuchsbarometers Technikwissenschaften (acatech/VDI 2009) gaben vor allem die technikbegabten Schüler an, dass sie Mädchen für weniger technikkompetent ansähen als Jungen. Diese negative Fremdeinschätzung verstärkt die ohnehin gering ausgeprägte Selbstwerteinschätzung der Schülerinnen im Hinblick auf die eigene Technikkompetenz. Zudem gaben mehr als zwei Drittel der befragten Studentinnen und Ingenieurinnen an, im Verlauf ihrer Ausbildung oder ihrer Karriere Diskriminierungen erfahren zu haben. Hinzu kommen die strukturellen Diskriminierungen durch ein geringerer Einkommen, geringere Aufstiegschancen und höhere Arbeitslosigkeitsrisiken.

Ein weiteres Merkmal der deutschen MINT-Bildungslandschaft ist das vielseitige und umfassende Angebot an außerschulischen Lernangeboten. Während sich in anderen Staaten Programme zur MINT-Vermittlung auf Science Center und nationale Museen konzentrieren (vgl. www.ecsite.com), kommt in Deutschland eine Vielzahl weiterer dezentraler außerschulischer Lernorte in Unternehmen und Kommunen hinzu (acatech/VDI 2009, Stiftung Haus der kleinen Forscher 2009, Renn/ Pfenning 2010a, b). Viele davon sind noch nicht evaluiert worden und, wo dies der Fall ist, zeigen z.B. die von der Universität Stuttgart und acatech gemeinsam durchgeführten Untersuchungen ein geringes $\mathrm{Ma} ß$ an systemischer Effizienz (acatech 2011). Dieses Defizit ergibt sich vor allem aus zwei Gründen: Es fehlt eine systematische Vernetzung und Verzahnung der Initiativen, und dadurch kommt es zu einem eher fragmentierten Angebot, das keine Kontinuität über den Zeitraum 
von der primären bis zur tertiären Bildung aufweist. Zudem kontrastieren diese oft attraktiv gestalteten außerschulischen Angebote mit den eher lehrerzentrierten und auf Wissensvermittlung ausgerichteten Unterrichtsangeboten in der Schule.

Darüber hinaus sind die Vielzahl der MINT-Berufe und die Charakteristika der Berufswelt den Schülern und Schülerinnen nicht bekannt. Eine Vielzahl neuer Disziplinen ist entstanden (z.B. Bionik, Bioverfahrenstechnik, Mechatronik, Gentechnologie, Umwelttechnik(en), Digitaltechnik, Softwareengineering u.v.a.), die bislang nicht im Bildungskanon reflektiert werden. Bereits offensichtlich ist die zunehmende Ausdifferenzierung in den Studienangeboten, die sich wiederum noch nicht in den Berufsbezeichnungen, wohl aber in den Stellenausschreibungen wiederfindet. Ebenso nachgewiesen ist die Ausweitung der Ingenieurtätigkeiten hin zu kundenorientierten Aufgaben (Service, Vertrieb, Produktionssektor).

Ein weiterer Grund für die Defizite in der MINT-Bildung in Deutschland ist in der Altersstruktur der Lehrerschaft zu sehen. Wie in keinem anderen europäischen Land ist die deutsche Lehrerschaft in MINT-Fächern überaltert (OECD 2008c). Zum einen sind dadurch die Unterschiede bei den MINT-Leitbildern zwischen Lehrkräften und Schüler/-innen entsprechend groß, zumal sich angesichts der schnellen Produktzyklen vieler technischer Anwendungen die Diskrepanz von „school science“ und „real science“ (Sjøberg/Schreiner 2005) vergrößert und sich die Schulbildung vom Alltag der Jugendlichen entkoppelt. Zum anderen hinkt der aktuelle Wissensstand der Lehrerschaft angesichts der rapiden Erkenntnisgewinne in einigen Natur- und Technikwissenschaften sowie in der Informatik den jeweiligen Entwicklungen hinterher. Dies trägt dazu bei, dass die Schüler/-innen die Lust an diesen Fächern verlieren.

Die hier nur kursorisch angeführten pädagogischen, didaktischen und technischen Defizite tragen alle zu der Erkenntnis einer mangelnden Attraktivität der MINT-Bildung in Deutschland bei. Dabei geht es nicht um mangelnden Willen oder um ein zu geringes persönliches Engagement vieler Lehrkräfte. Vielmehr sind es strukturelle und systemische Faktoren, die zu der unbefriedigenden Situation beitragen.

Strategien zur Deckung des MINT-Bedarfs

Angewandte Strategien im internationalen Vergleich

Vier Strategien zur Deckung des volkswirtschaftlichen Bedarfs an Fachkräften lassen sich aus der Vielzahl der OECD-Studien und wissenschaftlichen Literatur extrahieren: 
Anwerbung ausländischer Fachkräfte (Immigration, Braindrain/-gain): Diese Strategie setzt auf die Anwerbung auswärtiger Fachkräfte. Sie setzt voraus, dass das jeweilige Land bei den potenziellen Bewerbern als attraktives Einwanderungsland gilt. Hinzu kommen ökonomische Anreize beim Gehalt und den Arbeits- und Lebensbedingungen. Verallgemeinert gilt, dass Staaten, die viel Wert auf die Integration von Menschen mit Migrationsbiographien legen, auch besonders erfolgreich sind, auswärtige MINT-Fachkräfte zu gewinnen (OECD 2010c). Englischsprachige Länder sind hier eher erfolgreich als alle anderen. Die Einwanderungsstrategie verfolgen vor allem die USA und Großbritannien, zum Teil auch Frankreich.

Die Strategie, die Einwanderung von Fachkräften aus dem Ausland zu fördern, ist nur in begrenztem Maße steuerbar. Sprachliche Barrieren und historisch gewachsene Zusammenhänge von Einwanderungs- und Gastland (z.B. ehemaliger Kolonialstatus oder das britische Commonwealth) sind oft entscheidende Strukturmerkmale, die die Migration von qualifizierten Fachkräften beeinflussen. $\mathrm{Zu}$ dem spielen Möglichkeiten zur Familienzusammenführung und die Gastfreundschaft gegenüber Fremden eine große Rolle. Für Deutschland gibt es kaum positive Voraussetzungen, die es als Einwanderungsland attraktiv gelten lassen. Die überwiegend kritischen Erfahrungen mit der Greencard für Informatikexperten dokumentieren diesen Mangel an Attraktivität. Zudem sind Einwanderungs- und Wirtschaftsförderungspolitik in Deutschland nicht aufeinander abgestimmt.

Hohe Studierendenquoten und intensive Talentförderung (Akademisierung): Bei dieser Strategie geht es darum, durch Erhöhung der Studienanfänger auch die Zahl der Studierenden in den MINT-Fächern zu steigern. Sofern genügend Nachwuchs vorhanden ist und sich die Immatrikulationswünsche mehr oder weniger zufällig verteilen, sollten auch die MINT-Fächer davon profitieren. Darüber hinaus können interdisziplinäre Abschlüsse dazu beitragen den Absolventen mehrere Berufslaufbahnen auch in MINT-Berufe hinein offenzuhalten. Diese Strategie wird vor allem in den skandinavischen Ländern verfolgt. In Deutschland wird ebenfalls eine Erhöhung der Studierendenquote angestrebt, was jedoch bisher nur mit mäBigem Erfolg gelang. Die Studierendenquote in Deutschland ist leicht angestiegen und liegt knapp über den OECD-Durchschnitt. Allerdings sind die Studienangebote sehr fachspezifisch ausgerichtet und ermöglichen nur im geringen Maße branchenübergreifende Berufseinstiege.

Abstimmen von Bildungssystem und Arbeitsmarkt sowie die Vernetzung außerschulischer und schulischer Bildung: Diese Strategie umfasst den Versuch, durch ausdifferenzierte und sehr berufspraktische akademische Bildungsangebote mehr Nachwuchs im MINT-Sektor zu gewinnen. Das Bildungssystem ist entsprechend praxisnah ausgerichtet. Studium und Berufswahl sind eng miteinander verbunden, interdisziplinäre Komponenten sind dagegen weniger stark ausgeprägt. Voraus- 
setzungen hierfür sind eine frühe Talenterkennung undeine Förderung und Begleitung der jungen Menschen von der Schule bis zum beruflichen Einstieg. Zudem findet eine intensive Berufsorientierung bereits in der Schule statt. Dieses Modell findet sich vor allem in den Niederlanden, zum Teil auch in Deutschland (z.B. durch die Fachhochschulen und neuerdings durch die dualen Hochschulen bzw. ehemaligen Berufsakademien) und Frankreich. Ein Alleinstellungsmerkmal Deutschlands ist die ausgeprägte Bildungslandschaft außerschulischer MINT-Angebote. Diese kompensieren teilweise auch die Defizite in der MINT-Bildung an den Schulen. Diese Angebote dienen überwiegend der Talentförderung und damit der erfolgreichen Rekrutierung von Fachkräften (acatech 2011, Renn/Pfenning 2010a).

Aktive Öffentlichkeitsarbeit und Wissenschaftskommunikation (PUSH): Diese Strategie konzentriert sich in Bezug auf die MINT-Berufe auf die Verbesserung des Wissensstandes in der Bevölkerung. Im Mittelpunkt stehen Programme zum „Public Understanding of Science and Humanities“(PUSH). Ziel dieser Maßnahmen ist nicht nur die Förderung des Interesses an MINT-Fächern, sondern auch die Verbesserung der Allgemeinbildung hin zu einem soliden Grundverständnis von Wissenschaft und Technik (literacy). Dazu dienen Science Center, Wissenschaftsmessen, öffentliche Vorführungen, Fernsehsendungen und neuerdings auch Internetbildungsangebote. Die PUSH-Strategie ist vor allem in den USA, Großbritannien und den Niederlanden vertreten. Zunehmend wird sie aber auch in allen europäischen Ländern umgesetzt. Sie steht parallel zu den anderen Strategien.

Die Strategie der ausdifferenzierten Bildungsangebote in Kombination mit speziellen PUSH-Angeboten führt zu Erfolgen, wenn es um die Verbesserung der Wissenschafts- und Technikmündigkeit geht. Diese Strategie trägt aber wenig zur Talentförderung und zur Verbesserung der Attraktivität von MINT-Studiengängen bei. Auch Staaten mit einer ausgeprägten PUSH-Kultur wie die USA und Großbritannien sind von einem Fachkräftemangel und einem rückgängigen Interesse an MINT-Berufen und Studienfächern betroffen. Staaten, in denen das PUSH-Konzept erst später aufgegriffen, dann aber auf den aktuellen Stand der Lernforschung gebracht wurde, können beim Kompetenzerwerb und bei der Wissenschafts- und Technikmündigkeit deutlich messbare Erfolge verzeichnen, wie die Erfahrungen in Finnland, Schweden und Norwegen aufzeigen. Diese Aktivitäten bleiben jedoch auf individueller Ebene wirkungslos, wenn kein Anschluss an eine kontinuierliche Förderung und Vertiefung zur MINT-Bildung gegeben ist (Hiller 2010, acatech 2011). 


\section{Erfolgsaussichten der Strategien}

Keine der vier Strategien ist allein in der Lage, beide Ziele der MINT-Bildung in ausreichendem Maße zu erreichen. Vielmehr ist eine Kombination mehrerer Strategien gefragt. Wichtigste Erkenntnis aus den vielen Studien und aus dem Ländervergleich ist die Einsicht, dass einfache und auf einzelne Maßnahmen bezogene Lösungsversuche wenig bewirken werden. Das ist für viele frustrierend, die mit großem Engagement Initiativen und Programme zur Verbesserung der MINT-Bildung ins Leben gerufen haben. Aber die wissenschaftlich durchgeführten Evaluationen sprechen hier eine eindeutige Sprache. Nur mithilfe einer systematischen Verknüpfung vieler Reformen und Maßnahmen kann man sich dem angestrebten Ziel nähern. Was benötigt wird, sind:

- eine enge Verzahnung schulischer und außerschulischer Initiativen zu einem integrierten und vernetzten MINT-Bildungsangebot,

- eine frühzeitig beginnende und kontinuierliche MINT-Ausbildung vom Kindergarten bis zum Hochschulabschluss,

- eine altersgerechte und auf der Methode des „inquiry-based learning“ beruhende Vermittlung von MINT-Wissensbeständen,

- parallel laufende, sich gegenseitig ergänzende Aktivitäten zur Ausbildung einer allgemeinen Wissenschafts- und Technikmündigkeit und zur individuellen Talentbildung (mit besonderem Schwerpunkt auf jungen Frauen und Jugendlichen mit Migrationsbiographie) sowie

- ein lebendiger und gesamtgesellschaftlich wirksamer Diskurs über Chancen, Risiken und Folgen von Wissenschaft und Technik für Wirtschaft, Gesellschaft und Kultur.

Generell gilt: Je höher der Anteil der MINT-Fächer in der Schule insgesamt ausfällt, je stärker differenzierte Lernangebote vorliegen, und je mehr auf das Konzept des „inquiry-based learning“ zurückgegriffen wird, desto eher gelingt eine Bildung hin zur Wissenschafts- und Technikmündigkeit, und desto größer ist der Anteil von Studierenden in den entsprechenden Studiengängen (OECD 2009 c, 2010 b, g, h). Der Vergleich der Bildungssysteme in Europa zeigt, dass weniger strukturelle, formale Unterschiede als vielmehr die didaktischen Zugänge und die Kontinuität der Betreuung entscheidend für eine erfolgreiche individuelle MINT-Bildung sind. Die in den PISA-Leistungstests erfolgreichen skandinavischen Länder beginnen früh mit der Techniksozialisation, bilden die Erzieherinnen und Erzieher akademisch aus, beachten die Geschlechterrelationen bei den Bezugspersonen und betonen praxisorientiertes und projektbezogenes Lernen. Es ist eine wichtige Erkenntnis, dass ein didaktisches Konzept, das auf projektbezogenen, eigenständigen und kooperativen Lernweisen beruht, statistisch den größten Einfluss auf die indi- 
viduelle Wahl eines MINT-Studienfaches ausübt. In diesen Bereich der Didaktik und der modernen Lehrmethoden zu investieren, ist also in jedem Falle vorteilhaft und kommt beiden Zielen zugute.

Als zweiter Erfolgsfaktor ist die Präsenz der MINT-Thematik in allen Fächern, verbunden mit der Möglichkeit, neben den naturwissenschaftlichen Fächern auch ein Fach oder einen Schwerpunkt „Technik“ zu belegen, zu nennen (Pfenning 2010). Die Thematisierung von sozialen, wirtschaftlichen und kulturellen Folgen und Voraussetzung der MINT-Inhalte in Fächern wie Sozialkunde, Geschichte, Literatur und Kunst fördern das Interesse an diesem Thema und helfen bei der Ausbildung und Entfaltung von Urteilskraft und Kompetenz.

Zum Dritten ist eine kontinuierliche und auf individuelle Bedürfnisse bezogene Talentförderung von besonderer Bedeutung, um einerseits die Jugendlichen für das spätere Ergreifen von MINT-Berufen zu gewinnen und andererseits ein Lernumfeld zu schaffen, das gleichzeitig fördert und fordert. Wichtig ist ein frühzeitiges und dann aber auch kontinuierliches Angebot von der Primarstufe bis zur Hochschulreife.

Zum Vierten geht es um die Ausgestaltung der MINT-Bildung. Die bisherigen Ergebnisse der Forschung weisen darauf hin, dass der integrative Charakter der MINT-Fächer stärker zum Inhalt der Bildung gemacht werden sollte. Es geht also nicht darum, den Anteil von Physik, Chemie, Biologie, physikalischer Geographie und Technik in der Schule zu erhöhen, sondern die Gemeinsamkeiten der MINTFächer im Sinne eines basalen Verständnisses der Naturphänomene und deren Gestaltungsoptionen durch Technik zu betonen und auf dieser Grundlage einen „inquiry-based“-Ansatz des Lernens zu verfolgen. Die Zusammenfassung dieser Fächer unter dem MINT-Oberbegriff unterstreicht bereits ihren interdisziplinären Charakter. Zum einen geht es um ein Grundverständnis der „Grammatik“ der Natur, zum anderen um eine Einsicht in die Gestaltungsoptionen durch Technik. Diese interdisziplinäre Sichtweise ist bisher aber noch zu wenig konkret ausgearbeitet worden. MINT-Bildung erfordert ein neues integratives Unterrichtsmodell, bei dem die tradierten Naturwissenschaften, die neuen Technologien und die mathematischen Grundlagen synoptisch miteinander verbunden werden.

\section{Forschungsbedarf}

Die großen Forschungsinstitutionen und -organisationen auf nationaler und europäischer Ebene fördern die naturwissenschaftliche und technische Fachforschung (CERN, ISS u.v.a.). Weniger ausgeprägt ist die sozialwissenschaftliche Begleitforschung zur Förderung des technisch-naturwissenschaftlichen Nachwuchses wie auch zur offenen gesellschaftlichen Diskussion über Chancen und Risiken von Zu- 
kunftstechnologien. Die Wirksamkeit von MINT-Bildungsmaßnahmen und -methoden ist vor allem auf europäisch-vergleichender Ebene kaum untersucht. Es gibt nur wenige Projekte zur Didaktik und Pädagogik der MINT-Bildung (vgl. POLLEN/Science Now, Update), deren empirische Basis oft fragil ist. Ausnahmen bilden hier die ROSE-Studie und der Eurobarometer "Youth and Science“. Die neueren OECD-Studien leisten einen ersten erfolgversprechenden Ansatz, die individuellen Determinanten der MINT-Bildung exakter und valider zu erfassen. Immer mehr berücksichtigen sie soziologische oder pädagogische Faktoren wie soziale Herkunft, Lernkulturen, Sozialisationseffekte und Interaktion.

Die Perspektiven der betroffenen Schüler, Schülerinnen und Studierenden werden dennoch bislang nur in geringem Maße in der empirischen Bildungsforschung berücksichtigt. In Deutschland wurde dieses Defizit mit den Studien Nachwuchsbarometer Technikwissenschaften und Motivation durch Modellprojekte sowie einigen ergänzenden Studien zumindest ansatzweise ausgeglichen.

Um Bildungsangebote effektiv und effizient zu gestalten, ist eine professionelle Begleit- und Evaluationsforschung unerlässlich. Allzu oft werden neue Bildungsinitiativen ins Leben gerufen, ohne gleichzeitig eine Evaluierung der Effekte anzustreben. Darüber hinaus gibt es zu wenig öffentliche Förderprogramme, die eine vergleichende Evaluationsforschung der MINT-Bildung finanzieren. Schließlich fehlt es auch an einer effektiven Vernetzung der Begleitforscher. Dies könnte eine Aufgabe der wissenschaftlichen Akademien in Deutschland werden.

\section{Empfehlungen}

\section{Strukturelle Empfehlungen}

- Die Studierendenquote sollte weiter erhöht werden, um mehr Studierende für die MINT-Studiengänge zu gewinnen. $\mathrm{Zu}$ diesem $\mathrm{Ziel}$ sollten auch neue $\mathrm{Zu}$ lassungswege zum Studium außerhalb der allgemeinen Hochschulreife geschaffen werden. Hier sind in Deutschland die dualen Hochschulen als ein wichtiges Erfolgsmodell zu nennen und weiter auszubauen.

- Junge Menschen mit Migrationsbiographien sollten gezielt gefördert werden, weilgerade in Einwanderungsgruppen aus Gründen des sozialen Aufstiegs MINT-Berufe ein hohes Ansehen genießen und sprachliche Barrieren weniger bedeutsam erscheinen.

- Um den Anteil von Frauen in MINT-Fächern zu erhöhen, insbesondere in den klassischen Ingenieurdisziplinen, sollten Studienangebote mit sozialen Bezügen (Medizin, Optik, Gesundheits- und Umwelttechnik) sowie interdisziplinäre Angebote ausgeweitet werden. Strukturelle Diskriminierungen von Frauen (ge- 
ringere Aufstiegschancen, niedrigeres Gehalt) sind durch wirksame Gegenmaßnahmen auf der betrieblichen Ebene zu verhindern und ggf. gesetzlich zu sanktionieren. Zur Stärkung intrinsischer Motive bei MINT-interessierten Schülerinnen sind zeitlich begrenzte monoedukative Angebote wirksam und erfolgversprechend.

- Ebenso erforderlich sind attraktive Angebote zum beruflichen Wiedereinstieg der ca. 40.000 bis 60.000 graduierten Ingenieurinnen, die nicht im erlernten Beruf arbeiten.

- Die Vereinbarkeit von Familienplanung und Beruf ist für junge Männer wie Frauen ein wichtiges Anliegen. Die Unvereinbarkeit belastet aber vor allem die Frauen. Diese generelle Problematik ist nicht auf die MINT-Berufe beschränkt. Sie erfordert einen umfassenden Systemwandel im Personal- und Zeitmanagement von Berufen.

- Fort- und Ausbildung der erwerbstätigen Ingenieure ist eine wichtige Aufgabe der Unternehmen, um das vorzeitige Ausscheiden älterer Ingenieure aus dem Erwerbsleben zu verhindern.

\section{Empfehlungen zur Förderung der Wissenschafts- und Technikmündigkeit}

- Eine frühzeitige Förderung des Interesses an und der Neugierde auf Mathematik, Technik und Naturwissenschaften ist pädagogisch möglich. Frühzeitige Förderung bewirkt eine positive affektuelle Identifikation mit dem Stoff, ermöglicht erste Erkundigungen der umgebenden Natur mithilfe von alltagsnahem Wissen und fördert ein Interesse am Entdecken und Forschen.

- Kinder eignen sich die „Grammatik der Naturgesetze“ und die Prinzipien der Forschung (z.B. Versuch und Irrtum) bereits im Grundschulalter an. In diesem Alter muss man mit dem ,inquiry-based learning“ beginnen.

- Bildungsinstitutionen sind miteinander zu vernetzen, um Redundanzen zu vermeiden und Synergieeffekte zu erreichen. Dies schafft die Voraussetzung für eine kontinuierliche Wissenschaftssozialisation von der primären bis zur tertiären Phase. Dies gilt insbesondere für die Vernetzung außerschulischer und schulischer Lernorte.

- Angebote von Schulen oder Bildungsinstitutionen zu MINT-Themen für den Freizeitbereich sind zu fördern, weil damit gerade in der Pubertät Brüche in der MINT-Sozialisation vermieden und die intrinsische Motivation zusätzlich gefördert werden. Hierzu zählen dezentrale MINT-Labore, MINT-AGs und Teilnahme an Wettbewerben.

- Die Vermittlung des „,sozialen Sinns der MINT-Disziplinen“, insbesondere der Technik, erfolgt nur selten im Schulunterricht, aber gerade dies ist für MINT- 
interessierte Schülerinnen sehr bedeutsam. Diese Vermittlung schafft die Basis einer individuellen Beurteilung technischer Innovationen für Alltag, Freizeit und Berufswahl.

- Inner- und außerhalb der Bildungsinstitutionen sind gesellschaftliche Dialoge über die Chancen, Risiken und Folgen von wissenschaftlichen Erkenntnissen und technologischen Innovationen für die Gesellschaft zu führen, um auf der einen Seite die Bedingtheit unserer Kultur durch wissenschaftliche und technische Leistungen und auf der anderen Seite die Freiräume für die Ausgestaltung dieser Leistungen bewusst zu machen.

\section{Empfehlungen zur spezifischen Talentförderung}

- Eine erfolgreiche Talentförderung in MINT-Fächern erfordert eine neue praxisorientierte Didaktik sowie neue Lehrmethoden hin zu mehr selbstbestimmten Lernstilen für die Zielgruppen. OECD-Studien belegen hierfür die besten Lerneffekte (OECD 2009c).

- Die Interaktionseffekte zwischen Lehrkräften und Schüler/-innen sind bedeutsamer als die Wirkungen einer Hightech-Ausstattung mit technisch-naturwissenschaftlichen Laboren. Allerdings ist eine entsprechende Infrastruktur für die Talentförderung unverzichtbar, weil Technik und Naturwissenschaften zunehmend durch technische Medien vermittelt werden.

- Die Betreuung der Jugendlichen durch ein interdisziplinäres Fachteam muss im Mittelpunkt der MINT-Bildung stehen. Dazu sollte die akademisch-pädagogische Ausbildung der Lehrkräfte und Erzieherinnen bzw. Erzieher, einschließlich deren Fortbildung, verbessert werden. Hier sind die skandinavischen Länder vorbildlich.

- Monoedukative Angebote fördern das Interesse von Mädchen an MINT-Fächern, insbesondere in den Technikwissenschaften.

- Ein spezifischer Technikunterricht bzw. die Aufnahme von speziellen technikzentrierten Unterrichtseinheiten in den MINT-Fächern fördert nachweislich das Interesse an diesen Wissenschaftsdisziplinen. Zudem wird damit ein wichtiger Beitrag zur allgemeinen Technikmündigkeit geleistet.

- Die Hochschulen sollten im Rahmen der MINT-Studiengänge attraktivere Studienangebote anbieten. Dort, wo dies bereits gemacht wird, hat sich die Zahl der Studierenden stark erhöht. Zudem kommen interdisziplinäre Abschlüsse und erhöhte Praxisanteile im Studium sowohl den Anliegen der Studierenden als auch den Anforderungsprofilen der späteren Arbeitgeber entgegen. 


\section{Empfehlungen zur Didaktik und Gestaltung von MINT-Bildung}

- Entscheidend für den Erfolg der MINT-Bildung ist eine frühe und kontinuierliche Vermittlung, die auf das jeweilige Alter pädagogisch und didaktisch zugeschnitten ist und dabei eine Mischung aus phänomenologischen und alltagsnahen Lernformen umfasst. Interdisziplinäre Projekte und fächerübergreifende Unterrichtsgestaltung verstärken diese Entwicklung.

- Lernformen, die Schüler/-innen ein praxis-, projektbezogenes und eigenständiges Experimentieren und Lernen in MINT-Fächern ermöglichen, sind auszuweiten. Formale Lernweisen sind dabei nicht gänzlich zu ersetzen, sondern als ergänzende Unterrichtseinheiten einzubauen, vor allem wenn es um den Wissenstransfer zur Erklärung komplexer Zusammenhänge geht. Zum Verhältnis von selbstbestimmten und formalen Lernformen besteht aber weiterhin Forschungsbedarf.

- Alle Lernformen sollen auf die Förderung intrinsischer Motive bei den MINTinteressierten Schülern und Schülerinnen ausgerichtet sein. Eine erfolgreiche MINT-Bildung kann maßgeblich dazu beitragen, Schülerinnen und Schüler zur eigenständigen Beschäftigung mit Naturwissenschaften und Technik anzuregen. Deshalb sind kleine Lerngruppen, eigene Experimentiermöglichkeiten, eine gute Ausstattung der Labore und ein Selbstverständnis der Lehrkraft als Mentor und Betreuer nötig. Der Unterrichtung durch zwei Lehrkräfte pro Klasse (z.B. in Skandinavien) eignet sich gut für die Doppelrolle der Lehrkräfte als Betreuer und als Vermittler von Wissen.

- Die Forschungsergebnisse zeigen, dass Jugendliche in Deutschland überwiegendstereotype und oft an der Realität vorbeigehende Vorstellungen vom Berufsalltag der MINT-Berufe haben. Aus diesem Grund sollten frühzeitig und mehrfach im Verlauf der Schulzeit Praktika in Unternehmen angeboten werden. Diese Praktika sollten darauf ausgerichtet sein, den Jugendlichen die Berufswirklichkeit aufzuzeigen. Die Praktika sollten so attraktiv gestaltet sein, dass Jugendliche diese auch aus eigenem Antrieb in Anspruch nehmen.

- Experimentelle Praxisangebote und Projektarbeit durch schulexterne Anbieter sind als zusätzliche Stützen einer attraktiven MINT-Bildung anzusehen, sie könnenjedoch die MINT-Bildung in der Schule nicht ersetzen. Externe Angebote wie Schülerlabore oder Science Camps sind hervorragend dafür geeignet, latente Interessen für MINT zu wecken und die Neugierde auf MINT-Fächer zu stärken, sie bleiben jedoch meist folgenlos, wenn sie nicht integrativ an den laufenden Schulunterricht anknüpfen. 


\section{Empfehlungen für die Wissenschaftsinstitutionen}

- Für eine integrative MINT-Bildung ist es wichtig, die wissenschaftlich gebotenen Lernziele mit modernen Unterrichtsmethoden und altersgerechten Lernformen in Einklang zu bringen. An dieser Aufgabe können die wissenschaftlichen Akademien konstruktiv mitwirken, wie das Beispiel der französischen Akademie der Wissenschaften belegt. Gerade in den Akademien besteht der Anspruch, den interdisziplinären Diskurs zu pflegen und die Wissenskultur lebendig zu halten. Zudem ist dort der jeweilige fachliche Sachverstand vorhanden, um das Essenzielle vom Peripheren trennen zu können. Hier können interdisziplinäre Lehrmaterialien zur Erfahrbarkeit von Natur und Technik, aber auch zu ihrem kulturellen Sinngehalt, zu sozialen und wirtschaftlich verankerten Funktionen und zu einem modernen Wissenschafts- und Technikverständnis entwickelt werden.

- Es sollte Aufgabe der wissenschaftlichen Institutionen einschließlich der Akademien sein, Forschungsergebnisse in Bezug auf fachliche Erkenntnisfortschritte und lerntheoretische Einsichten aus der empirischen Bildungsforschung für die MINT-Bildung aufzuarbeiten und den Institutionen der Bildungsplanung und -vermittlung zur Verfügung zu stellen. Die Umsetzung dieser Erkenntnisse in die schulische Praxis muss vor allem über die Lehrerausund fortbildung an den Hochschulen erfolgen. Hierfür empfehlen sich Bildungskommissionen, die jeweils die Lehrpläne und Ausbildungsziele überarbeiten.

- Nach skandinavischem Vorbild sind konstruktive Evaluationen von Schulen sinnvoll, um eine nationale Best Practice von Unterrichtsweisen zu erstellen und diese systematisch in die Lehreraus- und -fortbildung zu integrieren. In diesen Evaluationsstudien sollten vorbildhafte Modelle, Projekte und Wettbewerbe, die im Rahmen der schulischen wie außerschulischen MINT-Bildung angeboten werden, auf ihre individuellen Lerneffekte und ihre Wirksamkeit für die Ziele der MINT-Bildung überprüft werden.

\section{Ausblick}

Die Debatte zur MINT-Bildung hat fast alle Industriestaaten erreicht. Dabei geht es inzwischen nicht mehr allein um den drohenden Fachkräftemangel, sondern um die Frage nach den Kompetenzen und Fertigkeiten, die Menschen in einer globalisierten Wirtschaft und in einer hoch differenzierten Gesellschaft benötigen, um den Herausforderungen im Alltag und im Lebensumfeld gewachsen zu sein und um an den kollektiven Debatten der jeweiligen Zeit aktiv mitzuwirken. Mit dem 
Konzept der Wissenschafts- und Technikmündigkeit wurde ein Konzept entwickelt, das als Leitbild für die weitere Reform der MINT-Bildung dienen kann. Junge Menschen sollen befähigt werden, kompetent und sachgerecht die Folgen wissenschaftlicher Erkenntnisse und technischer Neuerungen zu erkennen und aus dieser Kenntnis heraus sowie auf der Basis ihrer eigenen Werte und Präferenzen zu fundierten Urteilen zu kommen.

Die ROSE-Studie belegt eindrucksvoll die Bedeutung des kulturellen Kontextes für die Einschätzung von Wissenschaft und Technik durch die Bevölkerung (Sjøberg/Schreiner 2005). In Gesellschaften mit einer sich noch entwickelnden Volkswirtschaft auf Basis technischer Innovationen werden Wissenschaft und Technik deutlich positiver bewertet als in den Gesellschaften, in denen Wissenschaft und Technik bereits ein sehr hohes Niveau erreicht haben. Dieser Befund signalisiert, dass wissenschaftlicher und technischer Fortschritt für sich noch entwickelnde Volkswirtschaften nach wie vor von den meisten als Instrument der Verbesserung der eigenen Lebensverhältnisse angesehen wird, während dieser Fortschrittsgedanke in hoch technisierten Gesellschaften inzwischen als Selbstverständlichkeit betrachtet wird.

Daraus sind zwei Folgerungen abzuleiten. Zum einen dürfen Wissenschaft und Technik nicht nur als Werkzeuge für wirtschaftliche Zwecke vermittelt werden, da diese selbst wieder im gesellschaftlichen Diskurs umstritten sind. Zum anderen muss es darauf ankommen, die kulturelle Eingebundenheit von Wissenschaft und Technik in allen gesellschaftlich relevanten Zusammenhängen zu verdeutlichen.

Debatten über einen gesellschaftlich erwünschten und antizipierten Fortschritt sind also keine Luxusveranstaltungen von „übersättigten“ Wohlstandsbürgern, sondern wichtige Bestandteile einer lebendigen Kultur, die um die Bedingtheit ihres eigenen Wohlstandes weiß und darauf aufbauend die möglichen Optionen klug und vorausschauend abwägt. Der Einsatz vieler neuer Technologien (z.B. in der Bio- und Gentechnik) berührt ethische Grundfragen der Akzeptabilität. Diese nicht nur in elitären Kreisen, sondern auch in der breiten Bevölkerung zu reflektieren ist eine wichtige Aufgabe allgemeiner MINT-Bildung, die weit über den schulischen Bereich hinausgeht. Formate der Bürgerbeteiligung zu Risiken, Chancen und Akzeptanzbedingungen von innovativen Technologien sind daher ebenso Bestandteile einer modernen MINT-Bildung wie die Einrichtung von Schülercafés, Schülerparlamenten und anderen innovativen Formaten der interaktiven Vermittlung von Wissenschaft und Technik im sozialen, politischen und kulturellen Kontext. Vorbildhafte Programme finden sich in Großbritannien (GM-und NanoNation), in den USA (Amerika Speaks) und in Deutschland (Bürgerdialog „Energie“, das FUTURProjekt oderdas PUSH-Programm ,Wissenschaft im Dialog“).

Weiterhin muss MINT-Bildung sicherstellen, dass alle Jugendlichen, die ein besonderes Talent für MINT-Fächer mitbringen, frühzeitig und kontinuierlich ge- 
fördert werden, damit die wichtigste Ressource, die ein rohstoffarmes Hochlohnland wie Deutschland besitzt, nämlich das produktive Wissen, in ausreichendem Maße und in hoher Qualität für die Gegenwart und die Zukunft zur Verfügung steht. In diesem Zusammenhang gilt es vor allem, attraktive Bildungsangebote für junge Frauen und Jugendliche mit Migrationsbiographie zu schaffen, denn gerade in diesen beiden Gruppen gibt es viele Potenziale für den MINT-Nachwuchs, die in Deutschland noch weitgehend brachliegen.

\section{Literatur}

acatech (2009): Strategien zur Förderung des Nachwuchses in Technik und Naturwissenschaft. Handlungsempfehlungen für die Gegenwart, Forschungsbedarf für die Zukunft. Berlin/Heidelberg.

acatech/VDI (2009): Ergebnisbericht Nachwuchsbarometer Technikwissenschaften. München/ Düsseldorf.

acatech (2011): Monitoring von Motivationskonzepten für den Techniknachwuchs (MoMoTech). Reihe „acatech berichtet und empfiehlt“, Nr. 5. München/Berlin/Heidelberg.

Arnold, A./Hiller, S./Weiss, V. (2010): LeMoTech - Lernmotivation im Technikunterricht. Abschlussbericht zum Projekt Lernmotivation und Lerneffekte im Vergleich schulischer und auBerschulischer Lernorte (gefördert vom BMBF). Universität Stuttgart.

Bundesagentur für Arbeit (ibv/BfA 2007): Der Arbeitsmarkt für Akademiker/innen in Deutschland: Arbeitsmarktberichterstattung Naturwissenschaftlerinnen und Naturwissenschaftler. Nürnberg.

Bundesagentur für Arbeit (ibv, BfA 2009): Der Arbeitsmarkt für Akademiker/innen in Deutschland: Arbeitsmarktberichterstattung Ingenieurinnen und Ingenieure. Nürnberg.

Eckerle, K./Weidig, I./Limbers, J. (2002): Mittel- bis langfristiger Bedarf an Ingenieuren im deutschen Maschinen- und Anlagenbau. Impuls-Stiftung - Stiftung für den Maschinenbau, den Anlagenbau und die Informationstechnik/Prognos AG Basel/VDMA (Hrsg.). Basel.

Egeln, J./Heine, C. (2005 Hrsg.): Indikatoren zur Ausbildung im Hochschulbereich. Studien zum Innovationssystem Deutschlands, Nr. 4/2005. Hochschul-Informations-System (HIS)/Zentrum für Europäische Wirtschaftsforschung mbH (ZEW). Mannheim/Hannover.

Eurostat (2008a): Statistik kurz gefasst - Ältere Humanressourcen in Wissenschaft und Technik. Ausgabe 26/2008. Brüssel.

Eurostat (2008b): Statistik kurz gefasst - Frauen in Wissenschaft und Technik. Ausgabe 10/2008. Brüssel.

Fédération Européenne d'Associations Nationales d'Ingenieurs (FEANI) (2010): The European Engineers Publication. More Engineers for Europe. FEANI News Issue 6. Brüssel.

Frietsch, R./Gehrke, B. (2005): Bildungs- und Qualifikationsstrukturen in Deutschland und Europa. Studien zum deutschen Innovationssystem Nr. 3/2005. Fraunhofer Institut für Systemund Innovationsanalyse/Niedersächsisches Institut für Wirtschaftsforschung. Karlsruhe/Hannover.

Godfroy-Genin, A.S. (2010 Hrsg.): Prometea - Women in Engineering and Technology Research. Münster/Berlin.

Grelon, A./Stück, H. (1996): Ingenieure in Frankreich 1747-1990. Frankfurt am Main. 
Hampel, J./Schneider, M./Spurks, A./Sautter, A. (2003): Nachwuchsmangel im baden-württembergischen Handwerk. Stuttgart.

Hartmann, E./Kussmann, M./Scherweit, S. (2006): Technik und Bildung in Deutschland. Technikunterricht in den Lehrplänen allgemeinbildender Schulen. Eine Dokumentation und Analyse, zugleich VDI Report Nr. 38. Düsseldorf.

Heidenreich, M. (1992): Ingenieure und Techniker in Frankreich. Eine Fallstudie über die Verschulung technischen Wissens. Arbeitsbericht 77 des FSP. Universität Bielefeld.

Hiller, S. (2010): Ergebnisbericht zur wissenschaftlichen Evaluation der Forscherinnen-Camps. Interner Arbeitsbericht der Universität Stuttgart.

Hiller, S./Pfenning, U./Renn, O. (2008): Ergebnisbericht zur wissenschaftlichen Evaluation des IdeenParks 2008. Universität Stuttgart/ThyssenKrupp-AG Düsseldorf. Stuttgart/Düsseldorf.

Ihsen, S./Jeanrenaud, Y./Hantschel, V. (2009): Potenziale nutzen - Ingenieurinnen zurückgewinnen: Zum Drop-Out von Ingenieurinnen. TU München/Impuls-Stiftung Baden-Württemberg/VDMA Baden-Württemberg. Stuttgart/München.

Impuls-Stiftung - Stiftung für den Maschinenbau, den Anlagenbau und die Informationstechnik/ Prognos AG Basel/VDMA (2005): Qualitative Anforderungen an die Ingenieurausbildung und die künftigen Bachelor- und Masterstudiengänge. Frankfurt am Main/Basel.

Minks, K.-H. (2005): Kompetenzen für den globalen Arbeitsmarkt: Was wird vermittelt, was wird vermisst? In: Grüneberg, K./Wenke, I. (Hrsg.): Arbeitsmarkt - Elektrotechnik - Informationstechnik. Berlin/Frankfurt am Main.

OECD (2008a): Measuring Improvements in Learning Outcomes. Best Practice to Assess the Value-Added of Schools. Paris.

OECD (2008b): Higher Education to 2030. Vol.1. Demography. Paris.

OECD (2008c): Education at a Glance. OECD Indicators. Paris.

OECD (2009a): Creating Effective Teaching and Learning Environments. First Results from TALIS. Paris.

OECD (2009b): Evaluating and Rewarding the Quality of Teachers. International Practices. Paris.

OECD (2009c): Education Today - The OECD Perspective. Paris.

OECD (2010a): Education at a Glance. OECD Indicators. Paris.

OECD (2010b): Recognising Non-Formal and Informal Learning. Paris.

OECD (2010c): Closing the Gap for Immigrant Students. Paris.

OECD (2010d): Pisa 2009 Results: Overcoming Social Background, Vol.II. Paris.

OECD (2010e): Pisa 2009 Results: Learning to Learn. Vol.III.Paris.

OECD (2010f): Pisa 2009 Results: What Students Know and Can Do. Vol.I. Paris.

OECD (2010g): Pisa 2009 Results: What Makes a School Successful? Vol.IV. Paris.

OECD (2010h): Pisa 2009 Results: Learning Trends. Vol.V. Paris.

Pfenning, U./Hulpke, H./Beilborn, C./Wieneke-Toutaoui, I. (2002): Naturwissenschaften und Technik mangelhaft - Konsequenzen für Forschung, Wirtschaft und Technologie in Deutschland. In: VDI (Hrsg.): Gesellschaft und Politik. Technische Bildung in der Schule: PISA und die Zukunft von Forschung und Technologie in Deutschland, Politik-Dialog des VDI Berlin, 5. September 2002. Berlin/Düsseldorf.

Pfenning, U./Hiller, S./Renn, O. (2011): Frauen für Technik - Technik für Frauen. Zur Attraktivität von Technik und technischen Berufen bei Mädchen und Frauen. In: Wentzel, W./Mellies, S./Schwarze, B. (Hrsg.): Generation Girls' Day. Berlin. S. 124-158.

Pfenning, U./Schulz, M./Lorenz, N. (2003): Frauen und die Muse der Technik-oder: Ist Technik männlich? In: Heinrich, E.D./Rentschler, M. (Hrsg.): Frauen studieren Technik- BedingungenKontext- Perspektiven, Band 41. Aachen. S. 105-128. 
Pfenning, U./Renn, O./Mack, U. (2002): Zur Zukunft technischer und naturwissenschaftlicher Berufe - Strategien gegen den Nachwuchsmangel. Stuttgart.

Pfenning, U. (2010): Mehr Technikbildung, bitte! In: DIDACTA: Das Magazin für lebenslanges Lernen,.S. 7-14.

Renn, O./Pfenning, U. (2010a): Was machen Andere anders - was machen Andere besser? Wissenschafts- und Technikbildung in Deutschland und dem europäischen Ausland. In: BerlinBrandenburgische Akademie der Wissenschaften (Hrsg.): Die Akademie am Gendarmenmarkt 2010/11. Berlin. S. 49-54.

Renn, O./Pfenning, U. (2010b): Die Attraktivität technischer Studienfächer und Berufe. In JuniorIngenieur-Akademie. Ein Modellprojekt für die gymnasiale Mittelstufe zur Förderung des naturwissenschaftlich-technischen Nachwuchses. Bonn. S. 28-33.

Schölling, M. (2005): Soziale Herkunft, Lebensstil und Studienfachwahl eine Typologie. Frankfurt am Main/Berlin.

Sjøberg, S./Schreiner, C. (2005): Young People and Science. Attitudes, Values and Priorities. Evidence from the ROSE Project. Keynote Presentation at EU's Science and Society Forum 2005. Brussels, March 8-11, Session 4: Fostering diversity, inclusiveness and equality in science. http://ils.uio.no/forskning/rose.

Stiftung Haus der kleinen Forscher (2009): Philosophie, pädagogischer Ansatz und praktische Hinweise zur Umsetzung. Berlin.

VDE (2010): Ingenieurinnen und Ingenieure der Elektrotechnik/Informationstechnik. Trends, Studium und Beruf. Verband der Elektrotechnik Elektronik Informationstechnik e.V. VDEIngenieurstudie. Offenbach/Frankfurt am Main.

VDI (2007): Ingenieurmangel in Deutschland - Ausmaß und wirtschaftliche Konsequenzen. Studie erstellt vom Institut der Deutschen Wirtschaft im Auftrag des VDI. Düsseldorf.

VDI (2011): Ingenieurstudie Deutschland: Ergebnisbericht. Düsseldorf.

\section{Internetquellen}

http:///www.ecsite.com. Webseite des internationalen Zusammenschlusses der Science Center mit dem Schwerpunkt auf europäischen Science Center.

http://www.mintzukunftschaffen.de/das-mint-meter-20.html. Webseite des MINT-Meters des Instituts der Deutschen Wirtschaft zu Köln mit jeweils aktualisierten Angaben zum Fachkräftebedarf in MINT-Berufen. Köln. Zugriff am 17.8.2011

http://epp.eurostat.ec.europa/portal. Internetseite des Statistischen Amtes der Europäischen Kommission Eurostat.

www.tecnopedia.de. Internetportal mit Informationen zu Modellprojekten, Lehr- und Lernmaterialen und Experimenten zur MINT-Bildung. 